\title{
POLÍTICA DE PROPRIEDADE INTELECTUAL E TRANSFERÊNCIA DE TECNOLOGIA NAS UNIVERSIDADES: UMA PERSPECTIVA DO NIT DA UNIVERSIDADE FEDERAL DO RECÔNCAVO DA BAHIA
}

\author{
E. A. PIRES ${ }^{1 *}$ e C. M. A. T. QUINTELLA ${ }^{2}$ \\ 'Universidade Federal do Recôncavo da Bahia (UFRB) \\ ${ }^{2}$ Universidade Federal da Bahia (UFBA) \\ edilsonprppg@gmail.com*
}

Artigo submetido em outubro/2015 e aceito em novembro/2015

DOI: $10.15628 /$ holos.2015.3600

\section{RESUMO}

O presente estudo analisa a política de propriedade intelectual e da transferência de tecnologia (PI\&TT) sob a perspectiva do Núcleo de Inovação tecnológica da Universidade Federal do Recôncavo da Bahia (UFRB). A pesquisa bibliográfica, a análise de documentos de Gestão Institucional e a aplicação de entrevistas semiestruturadas com gestores da UFRB foram os métodos utilizados para a realização dessa investigação. Os dados indicam que a Universidade tentou estabelecer uma política de inovação desde 2006, mas não teve sucesso, se deparando com problemas como a falta de recursos humanos, de uma cultura de proteção dos direitos de propriedade intelectual e o distanciamento com o setor empresarial. Em 2014, apesar da criação de coordenadorias para atuar somente com a proteção das criações e a transferência de tecnologias, adicionalmente é preciso adotar outras estratégias para intensificar sua política de inovação como: a criação de uma Comissão de PI\&TT; a ampliação do quadro de funcionários com capacitação em PI\&TT e ações para intensificar a cultura de inovação entre os acadêmicos e empresas.

PALAVRAS-CHAVE: Propriedade Intelectual, Transferência de Tecnologia, Política de inovação

\section{INTELLECTUAL PROPERTY POLICY AND TECHNOLOGY TRANSFER IN UNIVERSITIES: A PERSPECTIVE OF OFFICE FOR TECHNOLOGICAL INNOVATION FEDERAL UNIVERSITY OF RECÔNCAVO OF BAHIA}

\begin{abstract}
This study looks at intellectual property policy and technology transfer (IP\&TT) from the perspective of technological Innovation Center at the Federal University of Recôncavo of Bahia (UFRB). The literature review, analysis of Institutional Management of documents and the application of semi-structured interviews with UFRB managers were the methods used to carry out this research. The data indicate that the University has attempted to establish a policy of innovation since 2006, but was unsuccessful, faced with problems like lack of
\end{abstract}

human resources, a protective culture of intellectual property rights, and the gap with the business sector. In 2014, despite the creation of coordinators to act only with the protection of the creations and technology transfer, in addition it needs to adopt other strategies to enhance their innovation policy as: the creation of a Commission of IP\&TT; the expansion of table employees with training in IP\&TT and actions to strengthen the culture of innovation among academics and businesses.

KEYWORDS: Intellectual Property, Technology Transfer, Innovation policy 


\section{INTRODUÇÃO}

A elaboração de políticas de Ciência Tecnologia e Inovação (CT\&I) ganha cada vez mais intensidade nos planos estratégicos de países, regiões, estados, municípios e Instituições de Ciência e tecnologia (ICT), objetivando fortalecer o desenvolvimento econômico a partir da criação e aprimoramento de tecnologias e sua transferência para o setor empresarial. No Brasil, principalmente a partir do início do século XXI, políticas de CT\&I ganharam maior atenção do Estado brasileiro (MATIAS-PEREIRA, 2011). Outras questões como a formação de redes de pesquisa e a aproximação entre empresas, governos e ICT passaram a integrar o plano estratégico de todas estas instituições. Destacam-se nessas relações o crescimento da participação acadêmica, da proteção de Propriedade Intelectual (PI) e do licenciamento de tecnologias para o seguimento empresarial e industrial.

A gestão da PI e a consolidação de políticas de inovação atrelada aos pilares clássicos da educação superior - ensino, pesquisa e extensão - foram intensificados nas universidades públicas brasileiras a partir da Lei 10.973, de 2 de dezembro de 2004 (Lei de Inovação Tecnológica - LIT) e sua regulamentação pelo Decreto 5.603, de 11 de outubro de 2005. A gestão dessas políticas de PI se caracteriza como um importante instrumento de apoio ao crescimento econômico do país e, "se fazem necessárias, especialmente para as universidades e os centros de pesquisas e para as indústrias, visto que nesse campo ocorre grande parte da criação e inovação tecnológica e cultural" (MATIAS-PEREIRA, 2011, p. 585).

No entanto, apesar das melhorias no sistema de inovação brasileiro, através da Lei de Propriedade Industrial, das diretrizes de Política Industrial, Tecnológica e de Comércio Exterior, da LIT, da Lei do Bem e da Política de Desenvolvimento Produtivo, do Plano de Ação de Ciência, Tecnologia e Inovação para o Desenvolvimento Nacional - 2007-2010, existem ainda barreiras para a implantação e gestão dessas políticas no Brasil, de forma que o país "[...] ainda não conseguiu desenvolver um sistema de administração pública de gestão de propriedade intelectual compatível com as exigências das demandas num mundo globalizado" (MATIAS-PEREIRA, 2011, p. 576). Conforme o autor, as dificuldades na administração dessas políticas não permitem que se cumpra seu papel de geração e estimulo à inovação.

Assim, também relacionado ao fortalecimento da inovação nas ICT, outras ações foram realizadas para intensificar a política de inovação no Brasil. Uma delas foi a criação do Fórum dos Gestores de Inovação e Transferência de Tecnologia (FORTEC), em 2006, organização de representação dos responsáveis nas universidades, institutos de pesquisa e instituições gestoras de inovação e pessoas físicas, por gerenciar as políticas de inovação e das atividades de PI\&TT e já conta com 204 NITs de todas as regiões do país (FORTEC, 2014). Na região Nordeste do Brasil, uma iniciativa de destaque se refere à criação da Rede de Núcleos de Inovação Tecnológica do Nordeste (Rede-NIT-NE), em 2005, e já conta com a colaboração de mais de 50 instituições, coordenando ações de proteção da PI e da TT, a fim de promover a inovação em todo Nordeste (REDE DE NÚCLEOS DE INOVAÇÃO TECNOLÓGICA DO NORDESTE, 2014). Nesse mesmo ano foi criado no Estado da Bahia a Rede de Propriedade Intelectual e Transferência de Tecnologia da Bahia (REPITTec), motivada pela política de CT\&I do Estado da Bahia, de forma a "contribuir para a integração e o fortalecimento da PI\&TT na Bahia, a fim de sustentar o sistema de inovação do Estado" (FAPESB, 2012). 


\section{A POLÍTICA DE INOVAÇÃO NAS ICT DO BRASIL}

O crescimento do fluxo de tecnologia e conhecimento entre a comunidade acadêmica e as empresas se intensificou com a promulgação do Bayh-Dole Act (Lei de Inovação Americana) nos Estados Unidos, em 1980, permitindo às universidades comercializar os direitos de patentes resultantes das pesquisas financiadas pelo governo, exemplo seguido posteriormente por outros países, como o Brasil a partir de 2004 com a publicação da LIT (DIAS; PORTO, 2013).

A LIT, cujo objetivo é "estabelecer medidas de incentivo à inovação e à pesquisa científica e tecnológica no ambiente produtivo, com vistas à capacitação e ao alcance da autonomia tecnológica e ao desenvolvimento industrial do país" (BRASIL, 2004), foi proposta a partir dos debates coordenados pelo Ministério da Ciência e Tecnologia (MCT) e da Academia Brasileira de Ciências, ao discutir o papel do conhecimento e da inovação na aceleração do desenvolvimento social e econômico do país, culminando na elaboração do "Livro Verde - Ciência, Tecnologia e Inovação: desafios para a sociedade brasileira" (VIEIRA, 2008). Esta lei foi criada mediante um papel de destaque do Brasil no cenário internacional de Ciência e Tecnologia, porém o país não havia conseguido progredir no número de depósitos de Patentes desenvolvidas por residentes no Brasil (MACHADO, 2013). Para Lotufo (2009), a LIT é a primeira lei que estimula a interação ICTEmpresa, especialmente nas ICT.

A LIT apresenta aspectos necessários para a criação de uma política de inovação nas ICT e empresas (CREUZ; SILVA, 2006) e permite elevar o nível de parcerias entre eles com vistas a estimular o processo de inovação e romper com o ciclo da dependência tecnológica do país (MATIAS-PEREIRA; KRUGLIANSKAS, 2005).

São cinco os grandes grupos de normas compreendidos pela LIT: a constituição de ambiente propício às parcerias estratégicas entre as universidades, institutos tecnológicos e empresas; estimulo a participação de ICT no processo de inovação; normas de incentivo ao pesquisadorcriador; incentivo à inovação na empresa e; apropriação de tecnologia (BARBOSA, 2011). Segundo o autor, esse conjunto de normas objetiva propiciar uma cooperação entre os atores do processo de inovação (aproximação ICT/Empresas) através de ações de incentivo ao trabalho criativo dos pesquisadores, ao envolvimento de empresas nacionais em projetos de pesquisa e desenvolvimento (P\&D), à apropriação de tecnologias e a sua transferência ou licenciamento para o setor produtivo.

A LIT também possibilita "autorizações para a incubação de empresas no espaço público e a possibilidade de compartilhamento de infraestrutura, equipamentos e recursos humanos, públicos e privados, para o desenvolvimento tecnológico e a geração de processos e produtos inovadores" (MATIAS-PEREIRA; KRUGLIANSKAS, 2005. p. 10). Também de acordo com os autores, os pressupostos estabelecidos pela LIT se direcionam a incentivar a inovação tecnológica e reconhece a importância de transformar C\&T e P\&D em novos produtos e processos ou melhorar o que já existe para alavancar a promoção de produtos inovadores no mercado nacional e internacional. Porém, os autores também ressaltam que algumas questões complexas não foram tratadas pela LIT como a criação de mecanismos para facilitar a conexão com as políticas industrial e tecnológica, e também a não existência de flexibilidade de gestão das instituições de pesquisa.

A partir da LIT as atividades direcionadas ao desenvolvimento econômico apresentaram um amadurecimento pelas universidades, evidenciadas pela aproximação com empresas, através de 
pesquisas conjuntas, licenciamento de patentes de titularidade das universidades e da gestão de spin-offs acadêmicos (AMADEI; TORKOMIAN, 2009). Segundo as autoras essa aproximação se caracteriza como uma estratégia fundamental para a inovação e, por isso, despertou uma discussão sobre a PI\&TT nas universidades públicas, pelas autoridades governamentais e gestores de políticas de inovação das universidades.

A gestão de uma política de inovação está ligada a atividade de pesquisa que resulta em novos conhecimentos, que podem se transformar em novas tecnologias comercializáveis (GARNICA; TORKOMIAN, 2009). Os autores destacam que as universidades são estimuladas a trabalhar com empreendedorismo, a fim de promover a pesquisa e extensão, visando viabilizar formas de apoiar o desenvolvimento econômico.

Portanto, devido à necessidade de gerenciamento de políticas de proteção da PI e gestão das inovações geradas nas ICT e do papel de escritórios de transferência de tecnologia nesse processo, a LIT estabelece no caput do artigo 16 a obrigatoriedade das ICT em "dispor de núcleo de inovação tecnológica, próprio ou em associação com outras ICT", para gerir sua política de inovação. (BRASIL, 2004).

\subsection{Núcleos de Inovação Tecnológica no Brasil}

Antes da LIT, a aproximação da universidade com o setor produtivo ocorria pela interação do próprio pesquisador e a criação de um Núcleo responsável pela gestão da inovação tecnológica era facultada à instituição (SOUZA, 2013). A obrigatoriedade de implementação dos NITs estabelecida pela LIT busca melhorar o processo de inovação no país através da aproximação das ICT com as empresas. Assim, Nunes (2010) destaca as principais funções dos NITs, classificando-as em três tipos: Interna, Externa e de Proteção. As funções internas estão relacionadas com as atividades e objetivos voltados para os pesquisadores da ICT à qual o NIT é vinculado, enquanto que as funções externas são os serviços e atividades prestados às empresas, institutos de pesquisa, setores do governo, pesquisadores externos e também atividades de marketing institucional e, por fim, as funções de proteção são objetivos relacionados à proteção da PI e das tecnologias desenvolvidas, licenças e à transferência das tecnologias e do conhecimento (NUNES, 2010).

Segundo Trzeciak e Pereira (2010), o NIT é um agente de transferência de tecnologia com o papel de atender as demandas do mercado transferindo desenvolvimento científico para as empresas, órgão público etc, de forma a provocar impactos positivos na sociedade.

Porém, a ausência de uma política de inovação implementada nas ICT é caracterizada por Rocca (2009) como a primeira barreira que os NITs teriam que enfrentar para cumprir com seus objetivos adequadamente. Portanto, o autor destaca que, ao ser criado nas suas respectivas instituições, o trabalho dos NITs destinava-se primeiramente a implantar e estruturar uma política de proteção da PI e gestão de inovações geradas pelos pesquisadores, principalmente do ambiente acadêmico.

Assim, fatores são importantes considerar nesse processo de criação e consolidação dos NITs, tais como a dificuldade de recursos que os NITs dispõem para se estruturar, a falta do quadro permanente de funcionários capacitados em gestão da inovação e PI e a resistência apresentada por muitos pesquisadores em cumprir os requisitos necessários para proteger sua $\mathrm{Pl}$, como, por exemplo, a não divulgação da invenção que atende aos requisitos de novidade, atividade inventiva 
ou ato inventivo e aplicação industrial, possibilitando o patenteamento, fruto da falta de uma cultura institucional de proteção da PI (ROCCA, 2009).

No entanto, Castro e Souza (2012) ao investigarem o papel dos NITs nas universidades brasileiras, baseando-se nos casos da Universidade de São Paulo, Universidade Estadual de Campinas, Universidade federal do Rio Grande do Sul e a Universidade Federal do Rio de Janeiro maiores depositantes de patentes entre as IFES do país, concluíram que os NITs estão desempenhando um papel na gestão da inovação das universidades e, apesar do trabalho desses núcleos encontrarem-se ainda em fase de estruturação se destacam na mediação da relação da universidade-empresa.

\subsection{Mecanismos para intensificar a proteção da PI\&TT em universidade brasileira.}

De acordo com Quintella e Torres (2012), uma vez que uma tecnologia ou um processo é desenvolvido, precisa-se realizar a transferência de Tecnologia para a empresa. Estabelecer uma cooperação entre as universidades e o setor produtivo pode auxiliar na busca de novas invenções, estimular o desenvolvimento de inovações e transferência de tecnologias (NIEDERGASSEL; LEKER, 2010 apud SILVA; KOVALESKI; GAIA, 2013).

O intercâmbio de ideias, habilidades, experiência (know-how) e conhecimento entre a base de ciência e a indústria, no contexto da TT, são vistas como uma parte vital da missão da pesquisa em uma organização, de forma que a TT tende a assegurar o retorno econômico e os investimentos em P\&D (CYSNE, 2005). Porém, a autora ressalta que "o conhecimento produzido por universidades e laboratórios de pesquisa é registrado com um formato e linguagem difíceis para as empresas decodificarem de modo a absorverem e utilizarem" (CYSNE, 2005, p. 69), tornandose uma grave limitação para a TT. Por isso, se destaca a necessidade de empresas realizarem investimentos e desenvolver estratégias e programas de educação e de desenvolvimento de habilidades, para transformar uma tecnologia em inovação. Ainda conforme a autora, uma estratégia que pode ser utilizada para que o conhecimento codificado se torne mais fácil de ser absorvido e, assim, mais útil às empresas, seria incluir na infraestrutura necessária para a TT, serviços de informação para dar suporte às atividades de TT e de informação científica e tecnológica.

Também são facilitadores no processo de TT: a existência dos NITs; a disponibilidade de recursos em projetos que contemplam a parceria universidade-empresas; as chances criadas pela LIT e a Lei do Bem; o fortalecimento de uma cultura voltada para TT por parte das empresas; as adequações jurídicas das universidades para dar suporte aos pesquisadores na parceria com empresas; centros de pesquisa cooperativa de universidade-indústria, parques de $P \& D$ e incubadoras de empresas (LINK, SIEGEL; BOZEMAN, 2006; CARVALHO; CUNHA, 2013).

Já González-Pernía, Kuechle e Peña-Legazkue (2013) asseguram que a infraestrutura tecnológica proporcionada pela universidade, associada à qualidade do seu corpo docente e redes relacionadas com a indústria e outros parceiros acadêmicos, parecem ser críticos na transferência de novas tecnologias para as empresas. Os autores defendem que a experiência da equipe dos escritórios de transferência de tecnologia, a existência de parques tecnológicos onde a universidade está localizada e o conhecimento acumulado via patentes, são fundamentais para aumentar os licenciamentos entre universidades e empresas. 
No Brasil, os escritórios de transferência de tecnologia, conhecidos atualmente como NITs (Núcleo de Inovação Tecnológica) são o organismo responsável pela TT entre ICT e empresa e, foram criados para promover um ambiente favorável a parcerias estratégicas entre as universidades, institutos tecnológicos e empresas (QUINTELLA; TORRES, 2012).

Sobre as tentativas de TT entre laboratório ou universidade e o setor industrial, Cysne (2005, p. 70) destaca que este se refere a um processo que envolve organizações e profissionaischave em cada organização, como, por exemplo, "a própria instituição de pesquisa, uma ou mais organizações usuárias durante o desenvolvimento e teste do produto, uma agência de transferência, uma agência de fomento pública, um receptor para a manufatura e uma agência de fomento privada". A autora ressalta ainda que a interação entre a universidade e a indústria tem necessidade da construção de pontes que favoreçam a ligação entre os dois setores, de forma que o serviço de informação caracteriza-se como um caminho desse entrosamento.

$\mathrm{Na}$ universidade, um primeiro mecanismo que pode ser adotado para intensificar a proteção da PI e a TT em universidade brasileira é a adaptação de experiências de sucesso de outras universidades, com modelos de estímulo à PI e a TT já consolidados pela comunidade científica.

Um estudo realizado por Silva (2008) identificou que nas universidades brasileiras que tem tradição em políticas de PI\&TT, os NITs seguem basicamente a mesma estrutura, qual seja:

[...] um Conselho Executivo, normalmente presidido pelo Reitor da Instituição, uma coordenação executiva e assessorias de suporte às questões referentes à Propriedade Industrial, contratos de transferência, assuntos jurídicos e auxilio aos pesquisadores em redação e acompanhamento de pedidos de patentes (SILVA, 2008, p. 85).

Outro estudo realizado por Castro, Jannuzzi e Mattos (2007) apud Closs et. al. (2012), no Escritório de Transferência de Tecnologia da Universidade Estadual de Campinas (UNICAMP), elencou alguns aspectos positivos do seu funcionamento como: a contratação de escritórios especializados em marcas e patentes; banco de patentes disponível para consulta e de fácil acesso na internet; experiência acumulada dos integrantes da equipe e a atuação em parceria com os pesquisadores.

Diversos estudos (FUJINO; STAL, 2007; GEOGHEGAN; PONTIKAKIS, 2008; SANTANA; PORTO, 2009) sublinham que dentre as principais dificuldades para os NIT exercerem suas funções, se destacam a falta de pessoal e a inadequação do financiamento do NIT, de forma que a superação dessas dificuldades é fundamental para a consolidação dos Núcleos. Deve se considerar ainda que a capacidade dos NITs para cumprir a sua missão está relacionada aos recursos que têm à sua disposição (GEOGHEGAN; PONTIKAKIS, 2008). Também, o espaço físico inadequado à realização das atividades do NIT é um ponto negativo ao processo de TT nas universidades públicas brasileiras (CLOSS et. al., 2012). No entanto, o ensino de PI na graduação contribuiria para a capacitação de recursos humanos para os NITs, indústrias e escritórios de patentes, contribuindo ainda para profissionalização da produção de patentes nas universidades (SINISTERRA et. al. 2013).

São identificados também outros mecanismos que podem assegurar a tutela dos direitos intelectuais nas universidades e devem ser adotados pelos NITs como: "assinatura do Termo de Sigilo, adoção do questionário de invenção e da celebração do Contrato de Autores e Inventores pelos membros da equipe de pesquisa" (ARAÚJO E. et. al. 2010, p. 8). 
Também é destacada pelo estado da arte a importância de se estabelecer parcerias estratégicas com empresas para promover o desenvolvimento tecnológico e facilitar a TT. Nessa perspectiva, Niedergassel e Leker (2010) apud Silva, Kovaleski e Gaia (2013), enfatizam que a cooperação universidade-empresa, traz estímulos importantes para o desenvolvimento de inovações e transferência de tecnologias, ao auxiliarem na busca de novas invenções.

O estimulo ao empreendedorismo acadêmico também é visto como uma importante estratégia de TT de modo que se caracteriza como uma ação de aproximação do setor empresarial. Arbix e Consoni (2011) sublinham que os NITs também devem propor e motivar ações que aproximem a universidade com a atividade empresarial, como por exemplo, ações de empreendedorismo tecnológico e de ambientes de inovação, incluindo as incubadoras e parques tecnológicos e também Silva (2008) destaca que os NITs já atuam no apoio e desenvolvimento de incubadoras de empresas, estimulando o empreendedorismo no corpo discente.

Considera-se ainda que as incubadoras de empresas de base tecnológica, associada ao fortalecimento da cultura empreendedora entre os alunos, e a criação de spin-off acadêmicos são facilitadores do desenvolvimento tecnológico e do lançamento de inovações no mercado.

De acordo com Silva (2008), as incubadoras de empresas nas universidades, podem aproximar a academia e o setor empresarial, por meio de parcerias com empresas incubadas, podendo também contribuir para manter os profissionais egressos da universidade na região.

"Os spin-offs acadêmicos (SOA) são um tipo de mecanismo de transferência de tecnologia que vem ganhando destaque, em que as universidades, que são um tipo de ICT, comercializam as suas pesquisas para a criação de uma nova empresa" (GARCIA; GAVA, 2012, p. 7). Destacam-se no desenvolvimento de produtos e serviços inovadores (CAMATTA et. al., 2012) e tem grande impacto no desenvolvimento econômico local (ARAÚJO M. et. al., 2005). É também um dos principais mecanismos adotados pelas políticas de transferência de tecnologia dos NITs, pois estes são considerados uma importante força motriz para restaurar as estruturas industriais (ALGIERI; AQUINO; SUCCURRO, 2013).

As decisões da administração central no tocante à sua política de inovação têm grande importância para estruturação e consolidação do NIT "uma vez que cabe à alta administração definir a política de operação dos escritórios e a sua estrutura organizacional, as quais terão impacto direto sobre a sua atuação" (FUJINO; STAL, 2007, p. 114).

\section{METODOLOGIA}

Nessa pesquisa, a estratégia utilizada para o estudo caracteriza-se quanto ao método, como estudo de caso, quanto à sua abordagem, como pesquisa qualitativa e quanto ao objeto, como pesquisa exploratória.

Utilizou-se a Pesquisa de Campo, efetivada por meio da técnica da entrevista semiestruturada, orientada por um roteiro, realizadas individualmente com o Reitor da UFRB, a Pró-reitora da Pró-Reitoria de Pesquisa, Pós-Graduação, Criação e Inovação da UFRB (PGCI) e com o gestor da Coordenação de Criação e Inovação da UFRB (CINOVA), utilizando gravador, mediante autorização dos entrevistados. A realização das entrevistas ocorreu na primeira semana de junho de 2014, nos ambientes da UFRB, com horário marcado com os entrevistados mediante contato realizado com antecedência. 
Também se realizou uma pesquisa nos relatórios de gestão do NIT, dos anos de 2007 a 2013, relatórios de Gestão da UFRB, entre 2006 e 2013 e, no Plano de Desenvolvimento Institucional (PDI/UFRB 2009-2014) e Relatórios Parciais de Avaliação, além da resolução que regula os direitos de PI na UFRB (Resolução CONAC 015/2008).

Os procedimentos utilizados para análise e interpretação dos dados foram: o exame documental de forma a identificar e analisar a política de PI e transferência de tecnologia na UFRB, a forma como se dá a gestão dessa política na universidade e a estrutura organizacional do órgão responsável pela gestão dessa política (a CINOVA), servindo, ainda, como alicerce para a elaboração do roteiro das entrevistas; a transcrição das entrevistas, pelo pesquisador, ocorrendo concomitantemente a aplicação das mesmas; a classificação do conteúdo das entrevistas a partir do relato dos sujeitos entrevistados, dos assuntos abordados, do tema da pesquisa e da relevância da abordagem para o objeto de análise da pesquisa.

A discussão da pesquisa se baseou nos resultados apreendidos a partir análise de conteúdo das entrevistas e da análise documental, correlacionando com a abordagem conceitual que compõe o referencial teórico no tópico 2, anteriormente apresentado, permitindo investigar e discutir as estratégias de PI \&TT nas universidades, sob a perspectiva do NIT UFRB.

\section{A POLÍTICA DE INOVAÇÃO NA UFRB}

\subsection{Uma interpretação da política oficializada em documentos internos}

Um importante instrumento de análise da política de inovação das universidades são os documentos oficiais como os Relatórios de gestão, o Plano de Desenvolvimento Institucional (PDI), as resoluções etc.

No primeiro ano da UFRB, o relatório de gestão de 2006 já ressaltava que a Universidade, por meio da PRPPG, se esforçava para consolidar a pesquisa científica de qualidade, reconhecendo a capacidade científica e tecnológica de pesquisadores, professores e alunos. O relatório destaca que:

- A Coordenação de Ciência e Inovação Tecnológica, recentemente criada na estrutura administrativa da PRPPG, constitui uma iniciativa de fomentar e oferecer apoio à consolidação do resultado científico e tecnológico de excelente qualidade, agregando para o âmbito da academia modelos tecnológicos acessíveis e produtivos, promovendo um caráter empreendedor ao ensino, pesquisa e extensão.

- A dificuldade de implementação da CCIT tem sido a identificação de pessoal com perfil acadêmico para os principais núcleos (Propriedade Intelectual, Inovação Tecnológica, Polos Tecnológicos).

- Ressalta-se que todas as temáticas dos núcleos que compõem a CCIT são extremamente importantes e requerem a adequação pessoal capaz de criar no modelo da nova universidade (UFRB) uma cultura tecnológica e de proteção do conhecimento até então muitas vezes desconhecida e/ou pouco praticada.

- No entanto, algumas ações têm sido realizadas com colaboradores para conhecimento e implantação imediata do Núcleo de Propriedade Intelectual, mediante a participação em reunião técnicas e treinamentos em outras instituições. 


\section{$[\ldots]$}

- Foi solicitada inclusão do NPI da UFRB na Rede de Propriedade Intelectual e Transferência Tecnológica da Bahia (REPITTEC/SECTI-BA), que tem a atribuição de integrar e fortalecer a propriedade intelectual e a inovação tecnológica no SIB (Sistema de Inovação da Bahia) (UNIVERSIDADE FEDERAL DO RECÔNCAVO DA BAHIA, 2007, p. 33-34).

Essas ações demonstram que a gestão da UFRB já se preocupava com a inclusão de uma política de inovação que pudesse, além de fortalecer a produção científica, intensificar a produção tecnológica, disseminar uma cultura de proteção dos direitos de $\mathrm{PI}$ e inserir o empreendedorismo aos pilares básico das instituições de ensino superior: ensino, pesquisa e extensão. Porém, reconhece também que, dentre as dificuldades de consolidar um órgão responsável por gerir política de inovação da instituição, se destaca a falta de recursos humanos especializado em $\mathrm{PI}$ e Inovação Tecnológica (UNIVERSIDADE FEDERAL DO RECÔNCAVO DA BAHIA, 2007). Corrobora, portanto, com dificuldades encontradas em outras instituições, conforme já discutido por Rocca (2009).

Em 2008, a aprovação da resolução CONAC 15/2008 reflete novamente o interesse da universidade em estruturar uma política de inovação e $\mathrm{PI}$, dispondo sobre a propriedade e a gestão de direitos relativos à PI no âmbito da UFRB (UNIVERSIDADE FEDERAL DO RECÔNCAVO DA BAHIA, 2008).

Sobre as contribuições da Pós-graduação para essa política de inovação, de acordo com o PDI, está contemplado na política da pós-graduação na UFRB o incentivo à inovação tecnológica a partir do ensino de pós-graduação. Conforme o PDI, a política de ensino de pós-graduação da UFRB apresenta as seguintes diretrizes:

a) formar profissionais criadores, capazes de desenvolver novas técnicas e processos tendo em vista a expansão da indústria brasileira e as necessidades do desenvolvimento nacional em todos os setores;

b) transformar a universidade em centro criador;

c) formar professores qualificados para a expansão quantitativa do ensino superior;

d) promover o ensino de pós-graduação para a produção da inovação tecnológica (UNIVERSIDADE FEDERAL DO RECÔNCAVO DA BAHIA, 2009).

Essas diretrizes refletem muito claramente o entendimento dos gestores da UFRB no importante papel da universidade para contribuir com o desenvolvimento econômico e tecnológico nacional regional e local, principalmente através do desenvolvimento de novas tecnologias e processos com potencial inovador. Uma iniciativa de contemplar essas diretrizes se iniciou em 2011, com a criação da Especialização em Sociedade, Inovação e Tecnologia Social, ligada ao CCAAB e com 25 alunos matriculados (UFRB, 2012). O objetivo do curso é promover a formação de profissionais (gestores públicos, assessores, técnicos, dirigentes e lideranças de Empreendimentos Econômicos Solidários e de outras experiências coletivas) para atuar no processo de inovação, fomento, desenvolvimento, disponibilização e apropriação de Tecnologias Sociais e, tem como linhas de pesquisa, políticas públicas de ciência, tecnologia e inovação; elaboração, execução e avaliação de tecnologias sociais nos empreendimentos econômicos solidários; gestão de tecnologia social nos empreendimentos econômicos solidários (UNIVERSIDADE FEDERAL DO RECÔNCAVO DA BAHIA, 2014). 
No que se refere à política de pesquisa da UFRB, se destaca no PDI que o fortalecimento da pesquisa e da inovação tecnológica agrega valor aos processos, produtos e serviços produzidos no Recôncavo, fomentando intensamente o processo de inclusão social, de forma que os objetivos da pesquisa na UFRB são:

[...] desenvolver um conjunto de instrumentos que estimule a utilização do conhecimento gerado pela pesquisa aqui desenvolvida, de modo a produzir um crescimento econômico-sustentável; estimular a pesquisa científico-tecnológica e, a partir do conhecimento gerado, agregar valor a produtos, processos e serviços; estimular a inovação tecnológica entre os pesquisadores e despertar a consciência com relação ao importante papel da inovação para o aumento da competitividade da nossa economia; estabelecer uma diretriz para a pesquisa científico-tecnológica voltada para o desenvolvimento regional; estimular o acesso a recursos oriundos dos Fundos Setoriais, de modo que os pesquisadores disponham de recursos para o desenvolvimento de seus projetos e mapear toda a potencialidade de desenvolvimento científico e tecnológico, e estimular a pesquisa em áreas estratégicas (UNIVERSIDADE FEDERAL DO RECÔNCAVO DA BAHIA, 2009, p. 44-45).

Associado a esses objetivos, identificou-se que as competências da PRPPG se orientam para fortalecer, além da produção científica, a produção tecnológica e a apropriação de bens intangíveis relacionados aos direitos de PI. Dentre as competências da PRPPG, se relacionam diretamente com a política de inovação da UFRB: estimular a produção do conhecimento e a proteção das inovações criadas no âmbito acadêmico por meio da PI; coordenar a elaboração e acompanhar a execução de projetos de geração de tecnologia e inovação; coordenar e acompanhar a execução de projetos institucionais de pesquisa e inovação tecnológica (UFRB, 2009); avaliar as atividades de pesquisa e inovação tecnológica da UFRB; elaborar e implantar o plano estratégico de pesquisa e inovação tecnológica da UFRB; sugerir políticas para o desenvolvimento da pesquisa e inovação tecnológica da UFRB; coordenar a elaboração e acompanhar os projetos institucionais de pesquisa e inovação tecnológica; Estimular a produção do conhecimento e cadastrar a PI (UNIVERSIDADE FEDERAL DO RECÔNCAVO DA BAHIA, 2013).

Também relacionado a pesquisa, se destacam os contratos e/ou convênios firmados pela UFRB com outras instituições, com a preocupação de promover a inovação tecnológica, como, por exemplo, os convênios/contratos firmados com o CNPQ, o Instituto Federal de Sergipe (IFS) e o INSA (Instituto Nacional do Semiárido). Os objetivos desses convênios foram respectivamente: atuar em conjunto com o CNPQ para propiciar o atendimento da estratégia de governo na realização de projetos de pesquisas científicas, tecnológicas e/ou de inovação; ações no campo da educação, pesquisa e da inovação tecnológica na área de hidráulica e; acordo de cooperação técnica entre as partes, na promoção de trabalho de Pesquisa, desenvolvimento, inovação e articulação institucional no Semiárido Brasileiro (UNIVERSIDADE FEDERAL DO RECÔNCAVO DA BAHIA, 2013). 
4.2 A política de inovação na perspectiva dos gestores da UFRB ligados diretamente à inovação e à PI\&TT.

A PI\&TT na UFRB é analisada nesse tópico a partir da interpretação do conteúdo das entrevistas realizadas com os gestores da UFRB, envolvidos diretamente com as discussões e decisões sobre PI e transferência de tecnologia na universidade.

Uma primeira indagação feita aos entrevistados se referia a importância da PI nas universidades brasileiras. Os gestores afirmam que a PI assume o papel de proteger os resultados de pesquisa dos acadêmicos e a possibilidade de gerar recursos e novos negócios. Porém, afirmam que ainda é dada pouca importância para os direitos de PI, de forma que intensificar discussões sobre a proteção da PI no ambiente acadêmico e conseguir resultados significativos é um grande desafio, porque as universidades não foram preparadas para lidar com questões de $\mathrm{Pl}$, existindo um distanciamento da sociedade e do setor industrial.

Sobre a importância de disseminar a PI na UFRB, o gestor da CINOVA ressalta que a coordenação tem trabalhado exaustivamente, realizando cursos de redação de patentes e planejando o curso registro e proteção de cultivar e programa de computador, além de estar participando dos eventos realizados pela Pró-reitoria e promovendo palestras e outras ações para divulgação da PI. A Pró-reitora ressalta que a preocupação em disseminar a PI na UFRB já existe nos últimos anos, principalmente devido a exigência da LIT é necessária para que os conhecimentos gerados pelos pesquisadores sejam reconhecidos. Destaca ainda que a universidade tem trabalhando com o auxílio da FAPESB para que o tema seja disseminado e haja maior aproximação com os professores de forma que os produtos desenvolvidos sejam protegidos e se possam gerar inovações. Já o Reitor afirma que são muitos os desafios da UFRB, principalmente por ser uma universidade em implantação, com professores novos e que acabam ingressando o ambiente acadêmico sem uma cultura de proteção de direitos de PI. Ressalta que uma das ações para inserir essa cultura de PI é a criação de uma coordenação de inovação junto a Pró-Reitoria de Pesquisa e Pós-Graduação.

Sobre as ações de disseminação da PI, o gestor da CINOVA, destaca que já houve resultados significativos, pois muitos trabalhos já estão sendo preparados para serem protegidos sob os direitos de PI e patentes já foram depositadas, além das ações da CINOVA causarem um certo entusiasmo em alguns pesquisadores. Acredita também que com as ações que estão planejadas para o próximo semestre [segundo semestre de 2014] haverá um incremento no número de proteções.

Indagados sobre a política de PI na UFRB, os entrevistados destacam que não existe ainda uma política de PI consolidada na instituição, sendo que esta se encontra em fase de estruturação. Argumentam que a principal ação para fortalecer uma política de PI na universidade está relacionada à criação da CINOVA e a realização das atividades propostas para essa coordenadoria.

A gestora da PPGCl argumenta que uma das perspectivas com a implementação de uma política de PI na UFRB é que professores, pesquisadores e estudantes tenham conhecimento dos direitos de PI e da sua importância. Destaca ainda que, com a consolidação dessa política, os indicadores de produção tecnológica sejam aumentados e também, que a inovação possa ocorrer verdadeiramente na universidade. 
Sobre as expectativas após a criação da CINOVA, os gestores ressaltam que a criação de uma coordenação de inovação precisava ser feita devido à necessidade de se ter um órgão na universidade que desse maior visibilidade ao NIT, de forma que ações dessa coordenação possam alterar o cenário acadêmico e mudar a realidade da UFRB no que se refere à proteção dos direitos de PI, com maior participação dos professores na disseminação dessa cultura. Argumentam que essa mudança poderá ocorrer quando a cultura de PI for intensificada na universidade, associada também a um maior envolvimento das comunidades do Recôncavo. Porém, lembra o Magnífico Reitor que a autonomia das universidades públicas no Brasil prejudicou sua interação com o mercado e os meios de produção, sendo que, atualmente essa aproximação é um dos maiores desafios da universidade contemporânea.

De acordo com o gestor da CINOVA, criar apenas uma coordenação sem que seja disponibilizada uma infraestrutura adequada com a ampliação do quadro de funcionários e equipamentos não permite que os objetivos propostos sejam alcançados.

Além da infraestrutura, outras dificuldades que precisam ser superadas pela CINOVA são destacadas pelos entrevistados como: a pouca visibilidade da coordenação junto à comunidade acadêmica e não acadêmica, a falta de conhecimento sobre a importância de proteger resultados de pesquisas com direitos de Pl; a necessidade de qualificação da equipe, a implantação de uma legislação e uma política de PI mais agressiva que insira a cultura de PI na comunidade acadêmica e venha estender a necessidade de trabalhar a PI junto com a comunidade externa de forma que a universidade possa gerar produtos para beneficiar a sociedade.

Os mecanismos utilizados para superar essas dificuldades são citados pelos entrevistados como a realização de eventos para disseminar a cultura de PI, a realização de curso de capacitação de dois servidores e as tentativas de aproximar as empresas da universidade por meio da realização de um encontro chamado "Merenda Tecnológica".

Quando questionados sobre a inclusão do tema PI na graduação e na pós-graduação como um mecanismo de disseminação da PI na UFRB, os entrevistados afirmam que atualmente existem apenas algumas ações isoladas na disciplina Tecnologia de Alimentos e um módulo sobre inovação tecnológica ministrada pelo gestor da CINOVA. No entanto, os gestores acreditam que seria muito importante incluir nos cursos da universidade disciplinas para discutir direitos de PI não somente para ampliar o número de patentes, mas também para inserir uma cultura de proteção dos bens intangíveis, uma vez que o melhor caminho para inserir essa cultura é o aluno.

Sobre a regulamentação dos direitos de PI na UFRB, existe a resolução CONAC 015/2008 que regula os direitos de $\mathrm{PI}$ dos produtos, processos ou serviços gerados no ambiente acadêmico (UFRB, 2008), mas, segundo o gestor da CINOVA, esse instrumento legal precisa de atualização, pois não está adequado à realidade da UFRB, por ser muito geral e também, um instrumento desconhecido da comunidade acadêmica.

Os gestores informaram que já existe um esforço realizado pela CINOVA para atualizar essa resolução de forma a contemplar questões como a distribuição dos ganhos econômicos que forem gerados com os bens intangíveis protegidos por direitos de PI e também, a forma como é decidido o que deve ou não ser protegido. Conforme argumenta os entrevistados, atualmente não existe uma comissão especifica para tomar esse tipo de decisão, de forma que se protege o que é solicitado pelos pesquisadores, considerando apenas o critério de novidade, não havendo, por 
exemplo, um estudo prévio de viabilidade econômica, pois o objetivo principal na UFRB é ampliar o número de pedidos de proteção.

Como já mencionado, a CINOVA é o órgão responsável para gerir a política de PI da UFRB. Esta é uma estrutura nova e segundo o gestor desse órgão, apesar de ter sido aprovada há dois meses, sua criação ainda não está regulamentada e não houve um aumento no quadro de recursos humanos, mas a proposta é que seja composta pelos núcleos de: propriedade intelectual, transferência de tecnologia, criação e desenvolvimento tecnológico.

O gestor da CINOVA destaca que o principal objetivo da Coordenação é a estruturação de uma política de PI com a regulamentação da PPGCI, da CINOVA e a atualização da resolução de regulamentação dos direitos de propriedade intelectual na Instituição, além de atender as normas estabelecidas pela LIT.

Segundo os entrevistados, a CINOVA funciona em uma estrutura que, apesar de já ser um novo espaço físico, ainda é inadequada para desempenhar suas funções como, por exemplo, a falta de uma sala reservada para atender aos pesquisadores que surgem com novas $\mathrm{PI}$, de forma a garantir o sigilo das informações. Destacam também que não existe uma previsão orçamentária especifica para o órgão, havendo apenas a previsão de uma parte dos recursos da PPGCl para a execução de atividades básicas da Coordenação, mas que é muito limitada. Também não existe assessoria jurídica para dar assistência às atividades da Coordenação e o quadro de servidores é insuficiente. Entretanto, segundo os gestores, já se está empenhando esforços para ampliar o espaço físico, contratar novos servidores e qualificar os que trabalham na coordenadoria.

Os entrevistados, quando indagados sobre a existência da parceria universidade-empresa e a ocorrência de transferência de tecnologia para o setor produtivo, afirmaram que apesar de ser muito importante estabelecer a parceria com o setor empresarial/industrial, a comunidade acadêmica não percebe o valor dessa relação, de forma que ainda não existe uma aproximação consolidada entre esses sujeitos na UFRB, para a geração de produtos, processos ou serviços protegidos pelo direito de PI. Conforme os entrevistados a relação universidade-empresa, quando ocorre, é apenas através de alguns convênios estabelecidos para execução de projetos. Sublinham também que não há transferência de tecnologia entre a UFRB e empresas ou outras ICT.

Dentre os fatores que contribuem para o afastamento da relação universidade-empresa, são destacados pelos entrevistados: a diferença no ritmo de trabalho dos atores envolvidos nessa relação, onde a empresa é mais acelerada; o tempo em que uma tecnologia é gerada na universidade e a falta de uma cultura por parte dos pesquisadores na relação da academia com a empresa.

Os entrevistados também identificam algumas ações que podem diminuir a distância entres a universidade e a empresa de forma que é destacada a criação de mecanismos no sistema de produção, na legislação, na universidade e também, o estabelecimento de mais empresas no recôncavo e mesmo, um maior interesse de aproximação por parte dos pesquisadores e dos empresários.

Assim, estas ações estão relacionadas ao estimulo ao empreendedorismo, porém, os entrevistados afirmam que o estimulo ao empreendedorismo na UFRB é muito incipiente e não existe uma política institucional estabelecida para este fim. Conforme a Gestora da PPGCl estimular o empreendedorismo na universidade é importante, mas existe uma dificuldade de se construir 
uma cultura empreendedora porque até mesmo os alunos não estão interessados, de forma que é preciso primeiro trabalhar a valorização do empreendedorismo em toda a comunidade acadêmica. Ela ressalta que a PPGCI tem feito contato com o SEBRAE para trabalhar ações de estímulo ao empreendedorismo na UFRB, como a realização de cursos de capacitação. Já o Magnífico Reitor destacou que a inclusão do empreendedorismo e de uma cultura empreendedora é o grande desafio das universidades brasileiras e que poderá demorar gerações para se consolidar, porque, por muito tempo ações empreendedoras no ambiente acadêmico não eram vistas muito bem pela própria comunidade acadêmica, o que acabou provocando um distanciamento da sociedade e do setor produtivo.

A percepção dos gestores deixa claro que a UFRB não tem um planejamento preparado para estimular a comunidade acadêmica no desenvolvimento do empreendedorismo, de forma que os entrevistados sublinham que não existe nenhum tipo de infraestrutura para estimular alunos e professores a constituírem empresas e negócios inovadores. Um exemplo de infraestrutura que facilitaria esse desenvolvimento é citado nas entrevistas como a disponibilidade de uma incubadora de empresas de base tecnológica.

Sob a ótica da discussão dessa pesquisa, se verifica a necessidade de estabelecer ações de intensificação da PI e da TT na UFRB através do desenvolvimento e gestão de uma política de inovação com potencial inovador. Portanto, se propõe a seguir estratégias/mecanismos/ações que possam servir para as universidades brasileiras, principalmente a UFRB, consolidarem a proteção da PI e a TT na instituição.

1. Estratégias para intensificar a proteção de direitos de PI na UFRB

> Estimular a formação em PI na graduação e pós-graduação;

$>$ Mapear os projetos de pesquisa, classificar os de potencial inovador e assessorar os pesquisadores envolvidos nessas pesquisas quanto aos procedimentos para proteção dos resultados obtidos;

$>$ Prestar assistência aos pesquisadores na elaboração de novos projetos de pesquisa e nas formas de proteção de seus resultados;

> Estabelecer regras para publicação dos resultados de pesquisas com potencial inovador;

$>$ Adotar a utilização dos termos de sigilo e confidencialidade entre os membros das equipes de pesquisas com potencial inovador;

$>$ Oferecer cursos, minicursos, de proteção dos direitos de PI, redação de patentes, etc.;

$>$ Contratar empresas especializadas em redação de patentes para orientar os pesquisadores e redigir e acompanhar documentos de patentes;

2. Estratégias para intensificar a TT na UFRB

> Criar incubadoras de empresas de base tecnológica e estimular a formação de spin-off acadêmicos;

$>$ Estimular o empreendedorismo acadêmico;

> Identificar empresas interessadas em estabelecer parcerias com os pesquisadores da UFRB para a realização de pesquisas em conjunto;

$>$ Identificar empresas interessadas nos resultados de P\&D das pesquisas realizadas na universidade;

$>$ Realizar análise de mercado e viabilidade econômica de produtos e processo em potencial 
inovador;

Facilitar o acesso às patentes disponíveis para negociação;

$>$ Disponibilizar seu portfólio de produtos disponíveis para licenciamento;

3. Estratégias compartilhadas para intensificar PI\&TT na UFRB

$>$ Inserir paradigmas de PI\&TT e adotar experiências de sucesso de outras universidades brasileiras, com estruturação similar a UFRB e, com modelos de estímulo à PI\&TT já consolidados pela comunidade científica.

$>$ Criar um conselho executivo ou uma comissão de PI\&TT com participação do reitor e de professores e/ou técnicos administrativos;

$>$ Criar um regimento interno que normatiza as ações/funções da CINOVA junto à UFRB;

$>$ Criar uma equipe com servidores efetivos e especializados em PI\&TT para estabelecer um ambiente de trabalho estruturado e constante;

$>$ Disponibilizar assessoria jurídica especializada em PI\&TT;

- Estabelecer previsão orçamentária para atender as demandas do NIT;

$>$ Disponibilizar espaço físico adequado à realização das atividades do NIT;

$>$ Criar um plano estratégico que o direcione para o cumprimento do seu papel previsto na LIT, que é gerir as políticas públicas em CT\&l;

$>$ Sensibilizar a comunidade acadêmica (docente, discentes e técnicos administrativos) e possíveis parceiros externos em potencial para intensificar a potencialidade tecnológica, científica e socioeconômica na universidade e na região;

$>$ Atuar em parceria com outras ICT, governo e empresas.

\section{CONSIDERAÇÕES FINAIS}

A propriedade intelectual e a transferência de tecnologia nas universidades brasileiras se configuram ainda como uma barreira a ser superada, de forma que, mesmo 10 anos após a LIT, foram poucos os avanços no fortalecimento dos sistemas locais de inovação e, principalmente, na consolidação de uma política de inovação eficaz na maioria das ICT.

Estudos discutidos nesse trabalho mostram que, em sua maioria, as ICT não possuíam uma política de inovação implantada quando os NITs foram criados, caracterizando-se como a primeira barreira a ser superada pelos NITs, tornando mais tardia a mudança nos indicadores de produção tecnológica e transferência de tecnologia nas ICT.

Na UFRB se verificou que, apesar do seu empenho em estabelecer e promover uma política de inovação, é preciso considerar que essa política ainda não conseguiu "emplacar", necessitando do planejamento e estratégias que permitam promover uma política mais efetiva, que possa ampliar a interação da universidade com a indústria, o governo e outras ICT.

Em uma visão geral, os gestores entrevistados têm uma percepção compartilhada de que a cultura de proteção dos direitos de PI não está consolidada na universidade. Corroboram com o pressuposto de que intensificar a proteção do conhecimento no âmbito da UFRB se caracteriza como uma barreira a ser superada pela gestão da instituição, mas que os resultados que venham a surgir da superação desse desafio, são fundamentais para consolidar a universidade como 
instituição fortalecedora do desenvolvimento regional, ao transformar conhecimento em produtos e processos inovadores.

\section{REFERÊNCIAS}

1. brasileiras. Liinc em Revista, Rio de Janeiro, v. 8, no. 1, p 125-140. mar., 2012. Disponível em: http://www.ibict.br/liinc Acesso em: 25 jan. 2014.

2. CLOSS, L.; FERREIRA, G.; SAMPAIO, C.; PERIN, M.. Intervenientes na transferência de tecnologia universidade-empresa: o caso PUCRS. Rev. adm. contemp. [online], vol. 16, n. 1, p. 59-78, 2012. Disponível em: <http://www.scielo.br/scielo.php?script=sci_arttext\&pid=S141565552012000100005\&lng=pt\&nrm=iso>. Acessado em: 27 de fev. 2014

3. CREUZ, L. R. C.; SILVA, M.. A Lei de Incentivos à Inovação e à Pesquisa Científica e tecnológica. Revista IOB de Direito Administrativo, ano I, n. 6, p. 66-81, jun., 2006. Disponível em: <http://www.cv.adv.br>. Acesso em: 20 de dez. 2014.

4. CYSNE, F. P. Transferência de Tecnologia entre a Universidade e a Indústria. Enc. BIBLI: R. eletrônica de Bibl. Ciência da Informação, Florianópolis, n. 20, 2ㅇsemestre de 2005. Disponível em: <https://periodicos.ufsc.br/index.php/eb/article/download/1518.../315>. Acesso em: 10 jun., 2014.

5. DIAS, A. A.; PORTO, G. S. Gestão de transferência de tecnologia na inova Unicamp. Revista de administração contemporânea. Curitiba, v. 17, n. 3, jun., 2013. Disponível em $<$ http://www.scielo.br/scielo.php?script=sci_arttext\&pid=S141565552013000300002\&lng=pt\&nrm=iso>. Acesso em: 07 fev. 2014.

6. FUNDAÇÃO DE AMPARO A PESQUISA DO ESTADO DA BAHIA. Disponível em: <http://www.fapesb.ba.gov.br>. Acesso em: 25 out. 2013.

7. FÓRUM NACIONAL DE GESTORES DE TRANSFERÊNCIA DE TECNOLOGIA. Disponível em: $<$ http:// www.fortec-br.org/>. Acesso em: 05 jan. 2014.

8. FUJINO, A.; STAL, E. Gestão da propriedade intelectual na universidade pública brasileira: diretrizes para licenciamento e comercialização. Revista de Negócios, Blumenau, vol. 12, n. 1, p. 104-120, jan./mar., 2007. Disponível em: <http://www.uninove.br/PDFs/Publicacoes/cader nos_posgraduacao/cadernosv3n2adm/cdposv3n2adm_2d04.pdf>. Acesso em: 15 jan. 2014.

9. GARCIA, M. O.; GAVA, R.. Gestão da Propriedade Intelectual Como Suporte à Inovação Tecnológica: o Caso do Núcleo de Inovação Tecnológica da Universidade Federal de Viçosa. REDIGE, vol. 3, n. 03, dez. 2012. Disponível em: <http://www.cetiqt.senai.br/redige>. Acesso em: 25 fev. de 2014.

10. GARNICA, L. A.; TORKOMIAN, A. L. V. Gestão de Tecnologia em Universidades: Uma Análise do Patenteamento e dos Fatores de Dificuldade e de Apoio à Transferência de Tecnologia no Estado de São Paulo. Gest. Prod., São Carlos, vol. 16, n. 4, p. 624-638, out.-dez., 2009. Disponível em: <http://www.scielo.br/pdf/gp/v16n4/a11v16n4.pdf>. Acesso em: 03set. 2013.

11. GEOGHEGAN W.; PONTIKAKIS, D. From ivory tower to factory floor? How universities are changing to meet the needs of industry. Science and Public Policy, vol. 35, n. 7, p. 462-474, 2008. Disponível em: <http://spp.oxfordjournals.org.ez20.periodicos.capes.gov.br/content/ 35/7/462.full.pdf+html>. Acesso em: 27 fev. de 2014. 
12. GONZÁLEZ-PERNÍA, J. L.; KUECHLE, G.; PEÑA-LEGAZKUE, I.An Assessment of the Determinants of University Technology Transfer. Economic Development Quarterly, vol. 27, n. 1, jan. 2013. Disponível em: <http://edq.sagepub.com/content/27/1/6>. Acesso em: 09 mar. 2014.

13. LINK, A.; SIEGEL, D.; BOZEMAN, B. An Empirical Analysis of the Propensity of Academics to Engage in Informal University Technology Transfer. Rensselaer Working Papers in Economics. n. 610, 2006. Disponível em: <http://www.economics.rpi.edu/workingpapers/rpi0610.pdf>. Acesso em: 27 fev. 2014.

14. LOTUFO, R. A. A institucionalização de Núcleos de Inovação Tecnológica e a experiência da Inova Unicamp. In: SANTOS, M. E. R. dos; TOLEDO, P. T. M. de; LOTUFO, R. de A. (orgs.). Transferência de Tecnologia: estratégias para estruturação e gestão de Núcleos de Inovação tecnológica. Campinas - SP: Komedi, 2009.

15. MACHADO, S. P. Innovation policy and chemists education. Química Nova, São Paulo, v. 36, n. 6, 2013. Disponível em:<http://www.scielo.br/scielo.php?script=sci_arttext\&pid=S010040422013000600030\&lng=pt\&nrm=iso>. Acessos em: 07 fev. 2014.

16. MATIAS-PEREIRA, J. A gestão do sistema de proteção à propriedade intelectual no Brasil é consistente?. Revista de Administração Pública, Rio de Janeiro, v. 45, n. 3, Jun. 2011. Disponível em: <http://www.scielo.br/scielo.php?script=sci_arttext\&pid=S0034-761220110 00300002\&lng=en\&nrm=iso>. Acesso em: 03 Jul. 2014.

17. MATIAS-PEREIRA, J.; KRUGLIANSKAS, I. Gestão de inovação: a lei de inovação tecnológica como ferramenta de apoio às políticas industrial e tecnológica do Brasil. RAE - eletrônica, vol. 4, n. 2, jul./dez., 2005. Disponível em: <http://rae.fgv.br/eletronica>. Acesso em: 30 out. 2013.

18. NUNES, A. L. S.. Mudanças promovidas pela Lei da Inovação nas funções e práticas de gestão dos intermediadores da cooperação universidade-empresa das universidades federais. Dissertação (mestrado), Curitiba, PR: Universidade Federal do Paraná 2010. Disponível em: <http://www.dominiopublico.gov.br/download/texto/cp140785.pdf>. Acesso em: 28 out. 2013

19. QUINTELLA, C. M.; TORRES, E. A. Transferência de Tecnologia In: RUSSO, S. L.; SILVA, G. F.; NUNES, M. A. S. N. Capacitação em Inovação Tecnológica para Empresários. 2. ed. São Cristóvão: Edufs, 2012.

20. REDE DE NÚCLEOS DE INOVAÇÃO TECNOLOGIA DO NORDESTE. Disponível em: <Www.portaldainovacão.org>. Acesso em: 10 maio 2013.

21. ROCCA, E. Prefácio. In: SANTOS, M. E. R.; TOledo, P. T. M.; LOTUFO, R. A. (Orgs.). Transferência de Tecnologia: estratégias para estruturação e gestão de Núcleos de Inovação tecnológica. Campinas - SP: Komedi, 2009.

22. SANTANA, E.; PORTO, G. E agora, o que fazer com essa tecnologia? Um estudo multicaso sobre as possibilidades de transferência de tecnologia na USP-RP. Revista de Administração Contemporânea, vol. 13, n. 3, p. 410-429, 2009. Disponível em: <http://www.scielo. $\mathrm{br} /$ scielo.php?script=sci_arttext\&pid=S1415-

65552009000300005\&lng=pt\&nrm=iso\&tlng=pt>. Acesso em: 27 fev. 2014.

23. SILVA, J. C. U. Proposta de ação em propriedade intelectual em um Núcleo de Inovação Tecnológica sob a nova ótica estabelecida pela lei da inovação: Estudo de caso para a Universidade Federal da Paraíba. Dissertação (Mestrado em engenharia de Produção) Universidade Federal da Paraíba, João Pessoa, 2008. Disponível em: <http://www.livrosgratis. 
com.br/arquivos_livros/cp079943.pdf>. Acesso em: 26 fev. 2014.

24. SILVA, L. C. S.; KOVALESKI, J. L.; GAIA, S. Gestão do Conhecimento Organizacional visando à Transferência de Tecnologia: os desafios enfrentados pelo NIT da Universidade Estadual de Santa Cruz. Revista Produção Online, vol. 13, p. 677-702, 2013. Disponível em: <http://producaoonline.org.br/rpo/article/view/1314/1026>. Acesso em: 26 fev. 2014.

25. SINISTERRA, R. D. et. al. Panorama of the intellectual property, technology transfer and innovation for the Brazilian chemistry and comparison with the BRIC countries. Química Nova, São Paulo, vol. 36, n. 10, 2013. Disponível em: <http://www.scielo.br/scielo.php?script= sci_arttext\&pid=S0100-40422013001000008\&lng=pt\&nrm=iso>. Acesso em: 28 fev. 2014.

26. SOUZA, A. C. M. M. A importância do núcleo de inovação tecnológica para o desenvolvimento e científico tecnológico Instituto Federal Catarinense. 2013. Dissertação (mestrado profissional) - Universidade Federal de Santa Catarina, Centro Sócio-Econômico, Programa de Pós-Graduação em Administração Universitária, Florianópolis, 2013. Disponível em: <https://repositorio.ufsc.br>. Acesso em: 21 fev. 2013.

27. TRZECIAK, D. S.; PEREIRA, E. C. G. L. (Orgs.). Estruturação e Planejamento de Núcleos de Inovação Tecnológica. 2010. Disponível em: <http://inventta.net/wpcontent/uploads/2010/10/Manual_PlanejamentoNITs-FINAL.pdf>. Acesso em: 13 Fev. 2014.

28. UNIVERSIDADE FEDERAL DO RECONCAVO DA BAHIA. Relatório de Gestão 2006. Universidade Federal do Recôncavo da Bahia. Cruz das Almas - BA, 2007.

29. Resolução 015/2008, de 11 de dezembro de 2008. Dispõe sobre a propriedade e a gestão de direitos relativos à propriedade intelectual no âmbito da Universidade Federal do Recôncavo da Bahia, 2008.

30. Relatório de Gestão 2008. Universidade Federal do Recôncavo da Bahia. Cruz das Almas - BA, 2009.

31. Relatório de Gestão 2011. Universidade Federal do Recôncavo da Bahia. Cruz das Almas - BA, 2012.

32. Relatório de Gestão 2012. Universidade Federal do Recôncavo da Bahia. Cruz das Almas - BA, 2013.

33. . Disponível em www.ufrb.edu.br. Acesso em: 10 maio 2014.

34. VIEIRA, C. P.. Inovação tecnológica e desenvolvimento regional: as dimensões territoriais da lei de inovação tecnológica. 2008. Dissertação (Mestrado em Desenvolvimento Regional) Universidade Regional de Blumenau - FURB, Santa Catarina. Disponível em: $<$ http://proxy.furb.br/tede/tde_busca/arquivo.php?codArquivo=504>. Acesso em: 01 nov. 2013. 